\title{
SíNDROMES DE DISPERSIÓN DE SEMILLAS EN COMUNIDADES DE BOSQUE MESÓFILO DE MONTAÑA, EN LA REGIÓN CENTRO DE VERACRUZ, MÉXICO
}

\section{SEED DISPERSAL SYNDROMES IN MOUNTAIN CLOUD FOREST COMMUNITIES, IN THE CENTRAL REGION OF VERACRUZ, MÉXICO}

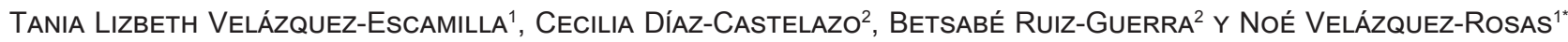

${ }^{1}$ Centro de Investigaciones Tropicales, Universidad Veracruzana. Xalapa, Veracruz, México.

${ }^{2}$ Red de Interacciones Multitróficas, Instituto de Ecología A. C. Xalapa, Veracruz, México.

* Autor de correspondencia: novelazquez@uv.mx

\section{Resumen}

Antecedentes: Los síndromes de dispersión permiten obtener patrones de dispersores potenciales entre comunidades, ambientes y taxones contrastantes, ayudan a entender su influencia en la composición, estructura y dinámica de las comunidades vegetales. Esta información es limitada en bosques mesófilos de montaña.

Preguntas: ¿Cómo se modifica la frecuencia de los síndromes de dispersión en función de la composición de especies leñosas y estratos de las comunidades vegetales? ¿Los síndromes de dispersión son útiles para describir los dispersores potenciales en el bosque mesófilo de montaña?

Sitios de estudio y periodo de investigación: El estudio fue realizado en tres comunidades de bosque mesófilo de montaña, en el centro de Veracruz (2014).

Métodos: Se determinaron los síndromes de dispersión de 64 especies en los tres sitios de estudio, utilizando características morfológicas de las diásporas y apoyo bibliográfico. Se comparó la frecuencia de los síndromes entre comunidades y estratos (sotobosque y dosel).

Resultados: Las frecuencias de los síndromes de dispersión no cambiaron entre comunidades, pero sí al interior de cada comunidad. La ornitocoria fue el síndrome más relevante, su importancia cambia con la densidad de las plantas y entre estratos. Con ayuda de información bibliográfica se encontró que $75 \%$ de las plantas identificadas con síndrome ornitócoro son dispersadas por aves.

Conclusión: La ornitocoria fue el síndrome más frecuente, su importancia se modifica en función composición, abundancia de las especies y estratos de la comunidad. Es necesario explorar la influencia de las aves en los patrones de distribución y reclutamiento de las plantas de estos bosques.

Palabras clave: Agentes de dispersión, estructura de la vegetación, frugivoría, ornitocoria, sinzoocoria.

\begin{abstract}
Background: Seed dispersal syndromes allow obtaining patterns potential dispersers between communities, environments and contrasting taxa, helping to understand the influence of dispersers on the composition, structure and dynamics of plant communities. This information is very limited in mountain cloud forests.

Questions: How is the frequency of seed dispersal syndromes modified according to the composition woody species and strata of plant communities? The seed dispersal syndromes are useful to describe potential dispersers in mountain cloud forests?

Study sites and dates: The study was carried out during 2014 in three mountain cloud forests communities in central Veracruz.

Methods: Seed dispersal syndromes of 64 different species plants were determined in the three study sites, based on the morphological characteristics of the diasporas and with bibliographic support. The frequency of the syndromes between communities and strata (understory and canopy) was compared.

Results: The frequencies of the seed dispersal syndromes did not change between communities, but they did within each community. Ornitochory was the most relevant syndrome, its importance changes depending on the density of the plants and the strata. With the help of bibliographic information, it was verified that $75 \%$ of the plants identified with ornithochory syndrome are dispersed by birds.

Conclusions: Ornitochory was the most relevant syndrome, although its importance was modified according to the composition and structure of the community. It is necessary to explore the influence of birds on the patterns of distribution and recruitment of the plants of these forests. Key words: Dispersal agents, frugivory, ornitochory, sinzoochory, vegetation structure.
\end{abstract}

This is an open access article distributed under the terms of the Creative Commons Attribution License CCBY-NC (4.0) international. https://creativecommons.org/licences/by-nc/4.0/ 
La dispersión de semillas es un proceso fundamental en el ciclo de vida de las plantas y es un elemento clave para entender sus patrones de distribución y abundancia (Dalling 2002, Levin et al. 2003, Carlo \& Morales 2016). Este proceso considera una serie de etapas desde que las semillas son removidas de la planta madre hasta su establecimiento (Wang \& Smith 2002). Además, participan distintos factores tanto bióticos como abióticos, que son los responsables de la movilidad y éxito en el establecimiento. Existen diversas herramientas que permiten conocer la naturaleza de las interacciones entre plantas, animales y vectores físicos involucrados en los procesos de dispersión, entre los más utilizados a nivel comunitario se encuentran los síndromes de dispersión (Howe \& Westley 1997, Loiselle \& Dirzo 2002).

Los síndromes de dispersión son un conjunto de características morfológicas que las plantas han desarrollado para facilitar la dispersión de sus semillas mediante vectores bióticos y abióticos (van der Pijl 1972, Guariguata \& Kattan 2002). El estudio de los síndromes de dispersión permite: i) inferir patrones de dispersión de semillas y realizar comparaciones entre ambientes y taxones, ii) entender la contribución de los diferentes agentes de dispersión en la dinámica del bosque y iii) hacer generalizaciones acerca de la influencia de los dispersores en la estructura y composición de vegetación (Howe \& Westley 1997, López \& Ramírez 1998, Loiselle \& Dirzo 2002, Deckers et al.2008, Chaín 2005, Buitrón-Jurado \& Ramírez 2014).

La hipótesis sobre la influencia de los animales como presiones selectivas para determinar las características de diseminación de los frutos ha sido un tema controversial; sin embargo, muchas investigaciones apoyan la hipótesis de que diferentes taxones de dispersores influyen en la evolución de las características de los frutos (Hampe \& Bairlein 2000, Lord et al. 2002, Lord 2004, Lamáscolo et al. 2008, Sinnott-Armstrong et al. 2018). En contraste, otras investigaciones han rechazado esta hipótesis, ya que no han encontrado una relación entre las características de los frutos y sus dispersores (Herrera 1989, Herrera 1992, Fischer \& Chapman 1993). Muchos de estos estudios se han basado en métodos filogenéticos comparativos con especies de climas templados (Herrera 1987, 1989, 1992) o del mismo género (Fischer \& Chapman 1993), dejando a un lado la variación intragenérica especialmente en taxa tropicales. Esta variación sigue siendo poco explorada en los análisis filogenéticos (Lomáscolo et al. 2008), por lo que el rechazo de la hipótesis de los síndromes de dispersión es más un reflejo de un problema metodológico que biológico (Jordano 1995). En las zonas tropicales las presiones selectivas bióticas tienen una mayor influencia; por ello, pueden incrementar el número de nichos y reducir su amplitud (Schemske et al. 2009), lo cual influye en las características reproductivas y de dispersión de las plantas (Rosas-Guerrero et al. 2014, Kuhlmann \& Ribiero 2016). De esta manera, los síndromes de dispersión pueden representar patrones de convergencia evolutiva para la adaptación a los grupos de dispersores más efectivos (Jordano 1995), tal como ha sido descrito para otros síndromes como los de polinización (Rosas-Guerrero et al. 2014).
Pese a las críticas, las investigaciones de los síndromes de dispersión han encontrado patrones relevantes entre la morfología de los frutos y los grupos de dispersores potenciales en distintos ecosistemas a diferentes escalas espaciales (Gentry 1982, Ganesh \& Davidar 2001, Vázquez \& Givnish 1998, Kuhlmann \& Ribiero 2016, Sinnott-Armstrong et al. 2018). En general, en hábitats secos es común encontrar diásporas dispersadas por viento, mientras que en hábitats húmedos son más frecuentes las diásporas dispersadas por animales (Gentry 1982, Howe \& Smallwood 1982). Además, existe una correlación positiva entre la precipitación y la frecuencia de zoocoria (Gentry 1982). Particularmente, en los bosques húmedos tropicales el $75 \%$ de las plantas producen frutos carnosos dispersados por frugívoros (Howe \& Smallwood 1982, Ganesh \& Davidar 2001, Buitrón-Jurado \& Ramírez 2014). En estos bosques se han encontrado diferencias entre los síndromes de dispersión y las distintas formas de crecimiento de las plantas, más del $90 \%$ de árboles y $75 \%$ de lianas tienen dispersión zoócora (Gentry 1982, 1995), principalmente por aves (Armesto \& Rozzi 1989). A lo largo de gradientes altitudinales se ha registrado que la zoocoria disminuye significativamente con el incremento de altitud (Cavieres et al. 1999). Esto puede ser resultado de la reducción de la temperatura ambiental y nutrientes del suelo, los cuales no favorecen la producción de pulpa en los frutos (Buitrón-Jurado \& Ramírez 2014, Chapman et al. 2016); así como, una disminución de vertebrados hacia mayores altitudes (Cavieres et al. 1999).

A pesar de que existen numerosos estudios en zonas tropicales, los síndromes de dispersión y su relación con la estructura y composición en comunidades de bosque mesófilo de montaña (BMM) ha sido un tema poco explorado (BarbosaAlbuquerque et al. 2006, Vázquez \& Givnish 1998, BaltazarHernández 2014). Estos bosques se localizan en las regiones montañosas donde las nubes se condensan causando lluvias abundantes, alta humedad y niebla, durante gran parte del año (Bruijnzeel et al. 2010). Su vegetación se caracteriza por presentar una elevada heterogeneidad tanto florística como estructural (Vázquez \& Givnish 1998, Velázquez-Rosas et al. 2002, Scatena et al. 2010, Williams-Linera et al. 2013, Toledo-Garibaldi \& Williams-Linera 2014).

El síndrome de dispersión que predomina en estas comunidades es la zoocoria, principalmente la endozoocoria (Vázquez \& Givnish 1998, Baltazar-Hernández 2014). En estos bosques muchas especies de la familia Solanaceae se dispersan por zoocoria, principalmente por ornitocoria (44\%) y quiropterocoria $36 \%$. La primera prevalece en zonas menos perturbadas y de mayor humedad, mientras que la segunda en zonas con mayor grado de perturbación y menor humedad (Barbosa-Albuquerque et al. 2006). La ornitocoria es un síndrome de dispersión importante para la regeneración de los BMM, ya que las aves dispersan principalmente semillas de especies pioneras, las cuales pueden competir eficientemente con pastos y helechos, que desencadenan el proceso de sucesión secundaria (Hernández-Ladrón de Guevara et al. 2012, Baltazar-Hernández 2014). Aunque existen patrones generales de dispersión en los BMM, no se conoce como se modifican en función de la composición y estructura de las 
comunidades, atributos que se modifican en escalas espaciales relativamente cortas (Williams-Linera et al. 2013).

La variación de la riqueza de plantas en los BMM en México está asociada a los cambios en las condiciones climáticas y topográficas de los diferentes pisos altitudinales (Williams-Linera et al. 2013, Toledo-Garibaldi \& Williams-Linera 2014). Sin embargo, no se ha encontrado una correlación entre la estructura de la vegetación y condiciones climáticas en estos ecosistemas (Williams-Linera et al. 2013, Toledo-Garibaldi \& Williams-Linera 2014). Esto sugiere que existen otros factores, como las interacciones bióticas (herbivoría, polinización, parasitismo por patógenos y dispersión de semillas, entre otras) que pueden influir en la distribución, composición y estructura de estos bosques. En este contexto, el presente estudio se enfocó en determinar los síndromes de dispersión de semillas de especies leñosas en comunidades de BMM, para conocer su variación e importancia relativa, así como su relación con la composición y estratos (sotobosque y dosel) de la vegetación. Además, debido a las críticas que tiene la utilización de los síndromes de dispersión, se evaluó su utilidad para la determinación del agente dispersor. El conocimiento de los dispersores potenciales en las comunidades de BMM nos permite aproximarnos a entender el papel de los agentes de dispersión en la composición, estructura y dinámica de estos bosques, así como el rol de la vegetación en la retención de biodiversidad (e.g., vertebrados). Esta in- formación permitirá establecer estrategias de restauración, manejo y conservación de estos ecosistemas, los cuales son considerados como prioritarios para la conservación (Hamilton et al. 1995).

\section{Materiales y métodos}

Sitios de estudio. Se seleccionaron tres sitios a diferentes altitudes de bosque mesófilo de montaña (BMM) del centro de Veracruz, localizados en las áreas montañosas del volcán Cofre de Perote (Figura 1). Estos sitios no tienen registro de actividad de extracción intensiva en los últimos 30 años y presentan composiciones florísticas y estructuras contrastantes (Velázquez-Escamilla 2016; Tabla 1). El BMM se distribuye entre los 1,200 y $2,700 \mathrm{~m}$ snm, entre las latitudes $19^{\circ} 30^{\prime}-19^{\circ} 45^{\prime} \mathrm{N}$ y las longitudes $96^{\circ} 47^{\prime}-97^{\circ} 01^{\prime} \mathrm{W}$ (Williams-Linera 2012). Actualmente, el BMM se encuentra en fragmentos localizados en barrancas con pendiente pronunciada, o está representado por remanentes aislados o conectados por campos agrícolas, cafetales, potreros y asentamientos humanos (Williams-Linera 2012).

El primer sitio está ubicado en la reserva Santuario del Bosque de Niebla del Jardín Botánico Francisco Javier Clavijero del Instituto de Ecología, AC, ubicado en la Ciudad de Xalapa, Veracruz. Este sitio tiene un intervalo altitudinal de 1,250 a 1,280 m snm, su precipitación anual total es de 1,500

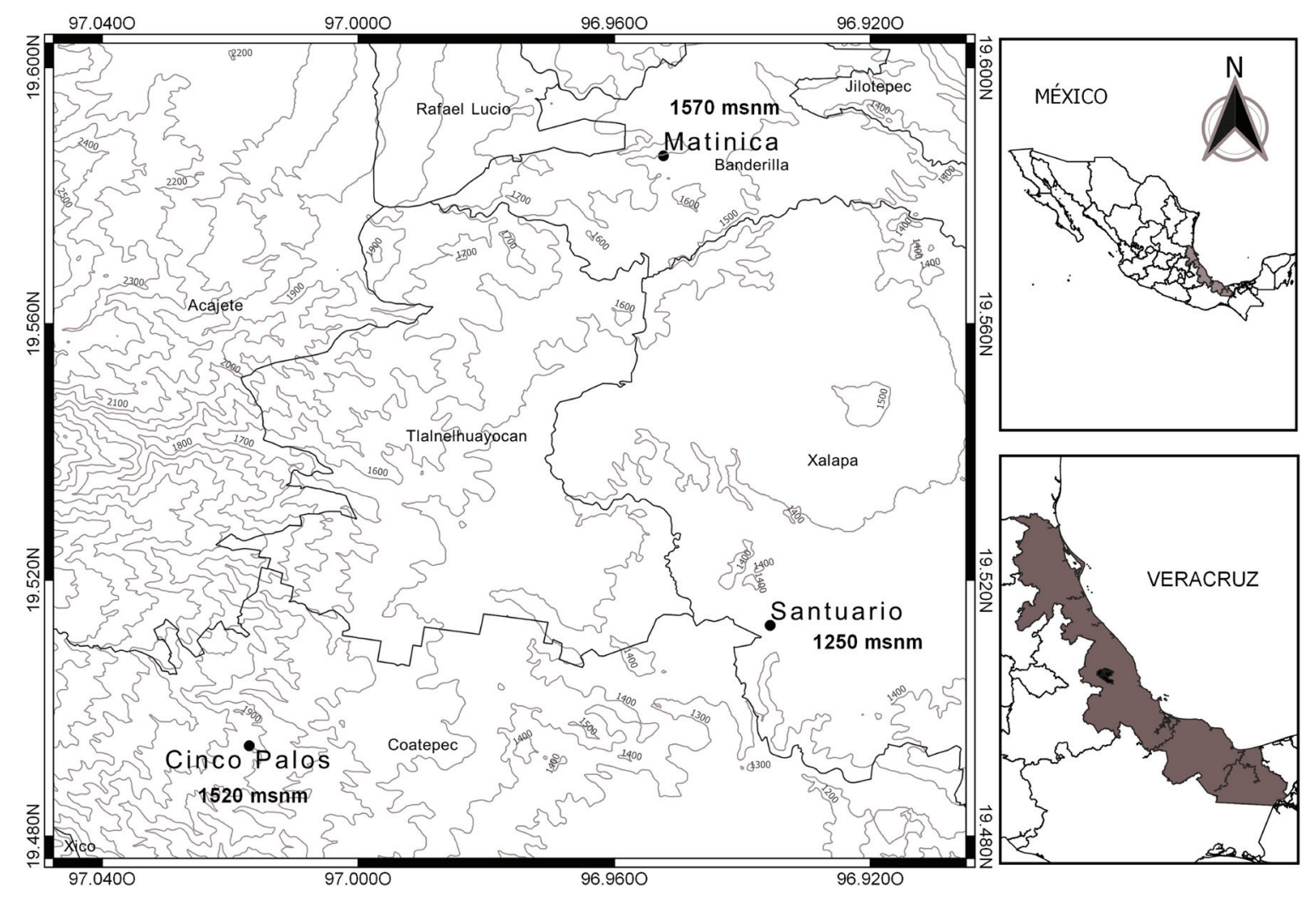

Figura 1. Localización geográfica de las comunidades de bosque mesófilo de montaña estudiadas. 
Síndromes de dispersión en el bosque mesófilo de montaña

Tabla 1. Riqueza y características estructurales de la vegetación de los tres sitios de estudio, de acuerdo con el análisis de estructural realizado por Velázquez-Escamilla (2016).

\begin{tabular}{lcccc}
\hline Sitio & $\begin{array}{c}\text { Altitud } \\
(\mathbf{m ~ s n m})\end{array}$ & Riqueza & $\begin{array}{c}\text { Densidad } \\
\left(\mathbf{i n d} / \mathbf{h a} \mathbf{a}^{-1}\right)\end{array}$ & $\begin{array}{c}\text { Área basal } \\
\left(\mathbf{m}^{\mathbf{2}} \mathbf{h a} \mathbf{a}^{-\mathbf{1}} \mathbf{)}\right.\end{array}$ \\
\hline Santuario del Bosque de Niebla & 1,250 & 32 & 3,520 & 36.9 \\
Cinco Palos & 1,520 & 26 & 2,730 & 80.3 \\
Martinica & 1,570 & 43 & 5,060 & 70.7 \\
\hline
\end{tabular}

y 2,000 $\mathrm{mm}$ y la temperatura media anual está alrededor de $18{ }^{\circ} \mathrm{C}$ (Williams-Linera 2012). Presenta una riqueza local de 32 especies leñosas y una densidad 3,520 ind ha $^{-1}$ con un diámetro a la altura del pecho (DAP; $1.3 \mathrm{~m})>2.5 \mathrm{~cm}$, de los cuales el $86 \%$ de los individuos se encuentran en estrato del sotobosque. Esta comunidad vegetal se encuentra dominada por las siguientes especies: Quercus germana, Psychotria galeottiana, Carpinus caroliniana y Quercus sartorii (Velázquez-Escamilla 2016).

El segundo sitio de estudio se localiza en la localidad de Cinco Palos, municipio de Coatepec, a una altitud de $1,520 \mathrm{~m} \mathrm{snm}$. La temperatura promedio anual es de $19.2^{\circ} \mathrm{C}$ y la precipitación promedio anual de 1,926 mm (Luna-Monterrojo 1997). Es un bosque dominado por Oreomunnea mexicana, especie que aporta casi el $79 \%$ del área basal total $\left(80.3 \mathrm{~m}^{2} \mathrm{ha}^{-1}\right)$. Otras especies relevantes son: Miconia glaberrima, Quercus cortesii y Zanthoxylum melanostictum. Presenta una riqueza de 26 especies y una densidad de 2,730 ind ha-1 con DAP $>2.5 \mathrm{~cm}$, aportada principalmente por árboles del sotobosque (Velázquez-Escamilla 2016).

El tercer sitio se localiza dentro del Área Natural Protegida la Martinica, en el municipio de Banderilla, en un intervalo altitudinal entre 1,570 y $1,620 \mathrm{~m}$ snm. Presenta una temperatura media anual de $18{ }^{\circ} \mathrm{C}$ y una precipitación pluvial media anual de 1,500 mm (Coordinación General del Medio Ambiente 2006). Este sitio cuenta con la mayor riqueza de especies (43 spp) y densidad de 5,060 ind $\mathrm{ha}^{-1}$. Aproximadamente el $50 \%$ del área basal total $\left(35.6 \mathrm{~m}^{2}\right.$ $\mathrm{ha}^{-1}$ ) de la comunidad es aportada por Quercus sartorii y Quercus lancifolia, árboles de dosel. Las especies más abundante en esta comunidad vegetal son Cinnamomum psychotriodes, Randia aculeata y Carpinus caroliniana (Velázquez-Escamilla 2016).

Determinación de sindromes de dispersión. Este trabajo utilizó los listados florísticos y estructurales obtenidos por Velázquez-Escamilla (2016), en el que se colocaron 10 cuadros de $10 \times 10 \mathrm{~m}$ en cada sitio y seleccionaron todas las especies leñosas con un diámetro a la altura del pecho (DAP) $\geq 2.5 \mathrm{~cm}$. En total se identificaron 32 especies en el Santuario, 26 en Cinco Palos y 43 en Martinica (Velázquez-Escamilla 2016). El total de especies estudiadas fue de 64 , debido a que varias se comparten entre los sitios.

Para la determinación del síndrome de dispersión se colectaron entre 20 y 50 frutos maduros (dependiendo de su disponibilidad) de tres o más individuos reproductivos mensualmente de junio 2014 a febrero 2015. De cada fruto se tomaron los siguientes datos: longitud, color, aroma, tipo (drupa, baya, nuez, aquenio, etc.) y se registró la presencia de estructuras especializadas para su dispersión (alas, vilanos, ganchos, comas, etc.). Debido a que no todas las especies fructificaron durante la época de colecta, sólo se obtuvieron diásporas de 31 especies en total; 16 especies en el Santuario, 12 en Cinco Palos y 14 en Martinica. El resto (33 especies) de las diásporas fueron caracterizadas con apoyo bibliográfico. La determinación del síndrome de dispersión se basó en la caracterización y categorización de Howe \& Westley (1997) y van der Pijl (1972). Estos autores caracterizaron los síndromes de dispersión utilizando un amplio número de caracteres morfológicos de las diásporas, esto permite predecir con mayor precisión a los agentes dispersores (Vázquez \& Givnish 1998). Además, para conocer la relación entre la estructura de la vegetación y los síndromes de dispersión las plantas fueron clasificadas por su distribución vertical en especies de sotobosque y dosel, las primeras fueron todas las que presentaron una talla entre 2 y $5 \mathrm{~m}$ en su fase adulta y las segundas fueron todas aquellas que rebasaron los $5 \mathrm{~m}$ de altura total.

Para evaluar la efectividad de los síndromes de dispersión en la determinación de los dispersores potenciales, se determinó si el síndrome ornitócoro registrado en este trabajo corresponde con los dispersores reportados en campo para las especies estudiadas. Para ello, se realizó una búsqueda bibliográfica de las especies con síndrome ornitócoro de las tres comunidades. Solo se utilizó al grupo de plantas dispersadas por aves debido a que este es el síndrome de dispersión más frecuente en los BMM.

Análisis estadísticos. Para establecer la importancia cuantitativa de los síndromes de dispersión en cada uno de los sitios de estudio y en los estratos de la vegetación se utilizó la herramienta multivariada de análisis de redes de interacciones. Se construyó una matriz de interacción representada por los síndromes de dispersión (nivel alto) y las especies vegetales (nivel bajo) presentes en cada comunidad. Posteriormente, para cada comunidad y estrato se determinó el valor de "species strenght" que establece la relevancia de cada nodo (síndrome de dispersión) en función de la abundancia absoluta de cada una de las especies que presenta cada uno de los síndromes, utilizando el paquete Bipartite 2.02 (Dormann et al. 2008, Dormann 2011) del lenguaje de programación R (R Core Team (2016). Posteriormente este valor se comparó entre comunidades y estratos con un modelo lineal generalizado con una distribución gamma y función de liga "inverse"; 
cuando se detectaron diferencias significativas se realizaron las pruebas de contrastes correspondientes, realizados con el lenguaje de programación R (R Core Team 2007).

\section{Resultados}

En total se identificaron siete síndromes de dispersión, de los cuales la exoozocoria solo se encontró en la comunidad de Cinco Palos (Figura 2A). La ornitocoria presentó el mayor número de especies (59), siendo Martinica la comunidad que registró el mayor número de especies con este síndrome $(58.7 \%)$. El segundo síndrome más frecuente fue la sinzoocoria (14.76\%), más representado en la comunidad de Cinco Palos (21.43\%). La quiropterocoria (11.76\%) fue mayor en el Santuario del Bosque de Niebla y la mamalocoria en la comunidad de Martinica (13.33\%). En cuanto a los síndromes de dispersión abióticos la anemocoria fue mayor en el Santuario y la autocoria en Cinco Palos (Figura 2A). En ambos estratos las especies zoócoras son las más frecuentes, en las tres comunidades el $14.6 \%$ de las especies de dosel se dispersan por sinzoocoria y el $12.3 \%$ por anemocoria y ornitocoria, mientras que en el sotobosque el $48 \%$ de las especies presentan ornitocoria (Figura 3).

La importancia relativa de los síndromes de dispersión; es decir, el valor de "species strenght" del análisis de redes, mostró que la ornitocoria es el síndrome más importante en cada una de las comunidades (Figura 2B). Los valores de "species strenght" no mostraron diferencias significativas entre sitios $\left(\chi^{2}=0.2\right.$, g.l. $\left.=1, p=0.306\right)$, pero si entre síndromes al interior de cada sitio $\left(\chi^{2}=15.3\right.$, g.1. $\left.=6, p<0.05\right)$. La ornitocoria fue el mecanismo de dispersión significativamente más relevante (tanto en términos de la abundancia de plantas que lo presentaron, así como en la frecuencia con la que ocurrió el síndrome) en las tres comunidades (Figura 2B).

Entre los estratos encontramos que en el sotobosque la importancia relativa de los síndromes presenta diferencias significativas $\left(\chi^{2}=1.5\right.$, g.l. $=8, p<0.05$; Fig. 3$)$. El síndrome más representado en este estrato fue la ornitocoria,
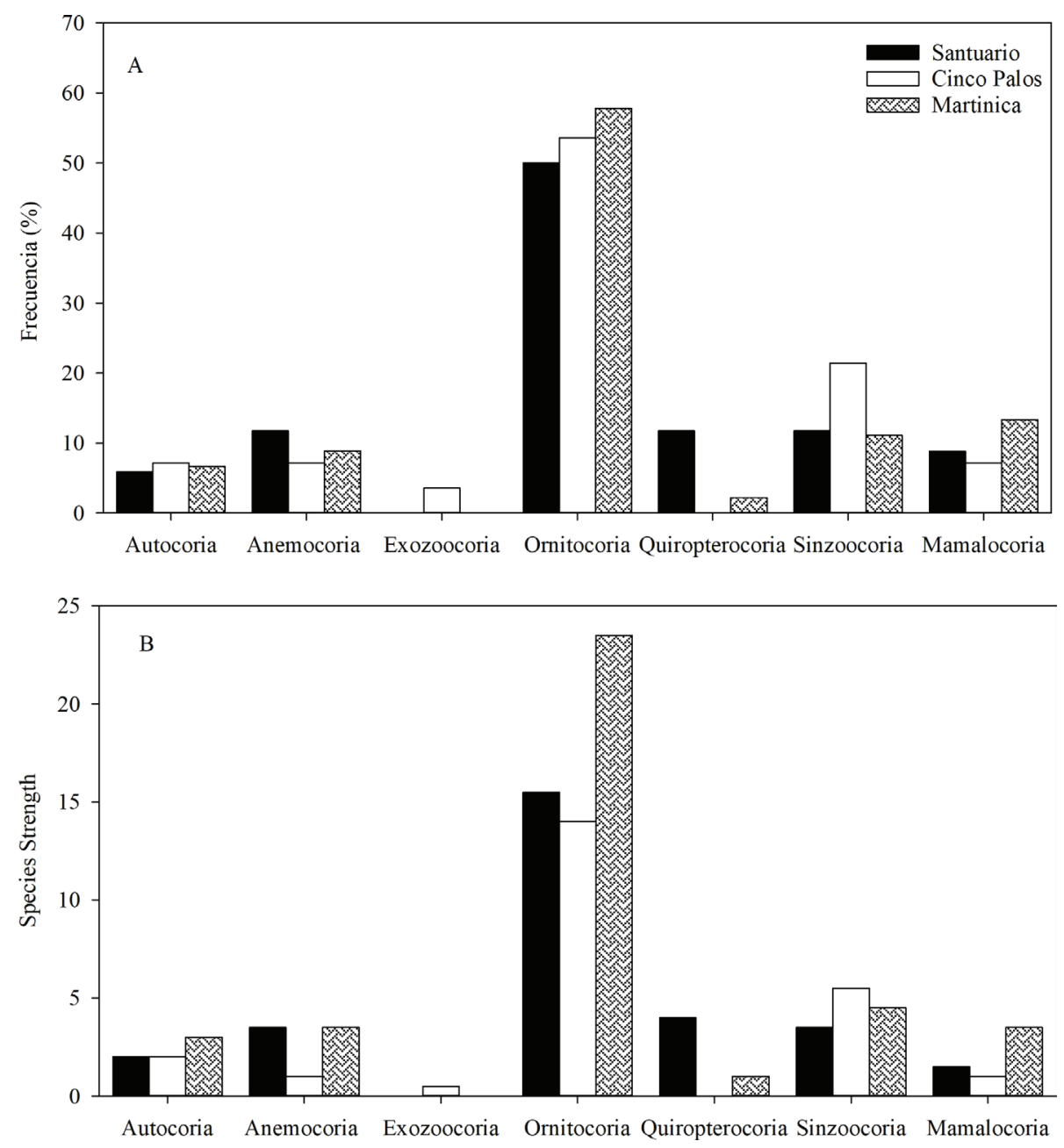

Figura 2. Frecuencia de los síndromes de dispersión (A) e importancia relativa medida en términos del parámetro "species strength" (B), en tres comunidades de bosque mésofilo de montaña en la región centro de Veracruz. 


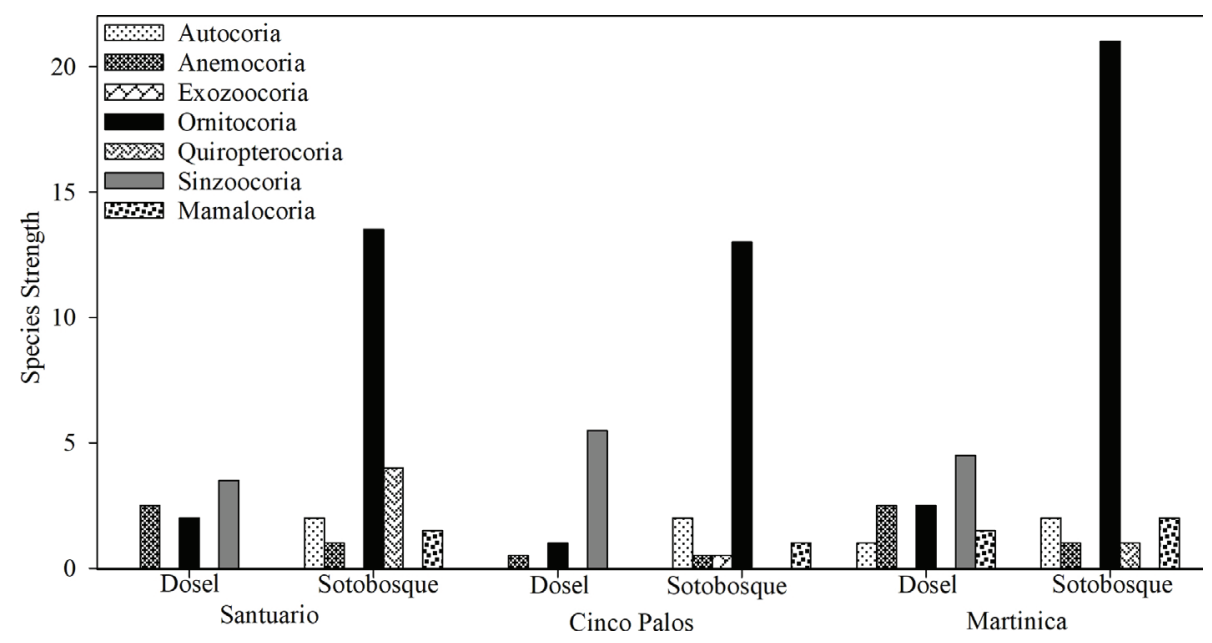

Figura 3. Importancia relativa de los síndromes de dispersión (species strength) en el sotobosque y dosel en tres comunidades de bosque mesófilo de montaña de la región centro de Veracruz.

presentando el valor más alto en la comunidad de Martinica; seguida por el Santuario y por último Cinco Palos (Figura 3). La quiropterocoria es otro síndrome importante en este estrato y es más frecuente en la comunidad del Santuario (Figura 3). En el dosel, la sinzoocoria es el síndrome con el valor más alto en las tres comunidades. La ornitocoria y la anemocoria también fueron importantes en el dosel, presentaron valores altos en las comunidades de Martinica y el Santuario; respectivamente (Figura 3); no obstante, no se encontraron diferencias significativas entre síndromes $\left(\chi^{2}=1.8\right.$, g.1. $\left.=5, p<0.02\right)$.

De las 41 especies determinadas con el síndrome de ornitocoria en este trabajo, en 31 se han identificado a las aves como su agente dispersor por distintas investigaciones (Figura 4). Para el resto de las especies no se encontró infor- mación bibliográfica sobre sus dispersores. Esta información confirma que el síndrome de dispersión ornitócoro permitió determinar al dispersor en más del $76 \%$ de las especies en cada sitio de estudio (Santuario del Bosque de Niebla [82 \%], Cinco Palos [87\%] y Martinica [77 \%]; Figura 4).

\section{Discusión}

Los resultados de esta investigación muestran que la composición de los síndromes de dispersión entre comunidades de BMM es similar, a pesar de que las tres comunidades presentan especies de plantas y estructura de la comunidad diferentes (Velázquez-Escamilla 2016). Sin embargo, la frecuencia e importancia de cada síndrome se modifican en función de la abundancia de las especies y los estratos

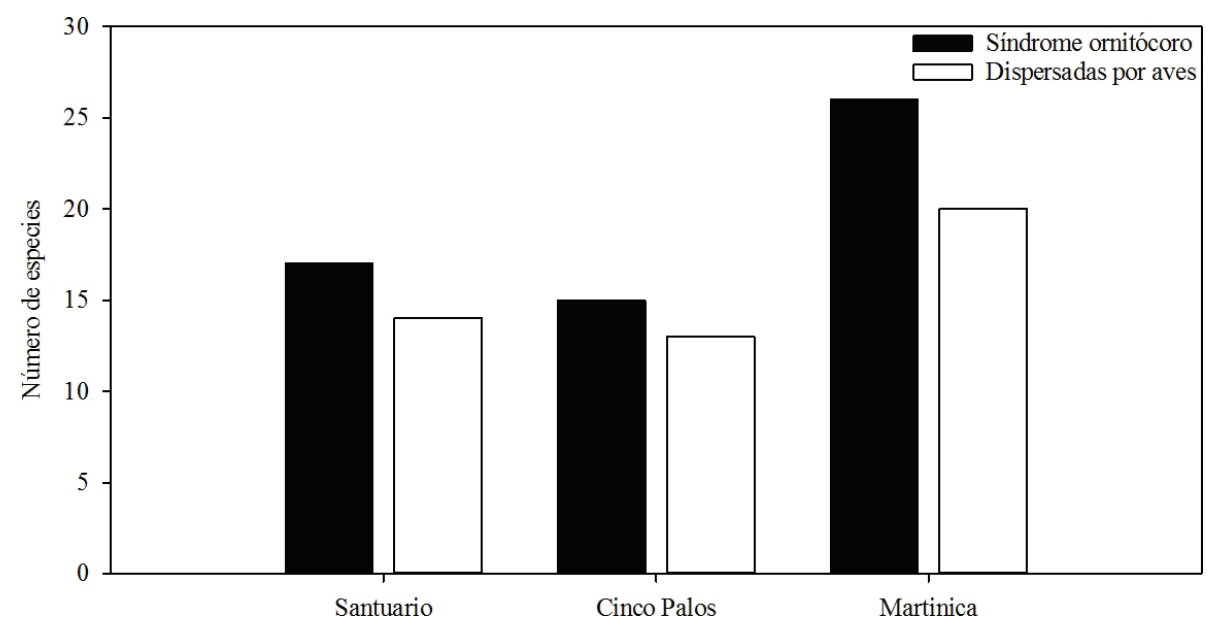

Figura 4. Número de especies con síndrome ornitócoro registradas en este estudio y especies que son dispersadas por aves de acuerdo con reportes de la literatura. 
de cada comunidad. La dispersión por vertebrados fue la más común en los tres sitios estudiados, principalmente la ornitocoria. Esto coincide con lo reportado en otros bosques húmedos del trópico, en donde el $90 \%$ de las plantas son dispersadas por animales (Howe \& Smallwood 1982, Gentry 1982, Wheelwright et al. 1984, Jordano 2000, Moles et al. 2007). Se ha propuesto que la elevada humedad en estos bosques favorece la frecuencia de plantas con frutos carnosos que son dispersados por vertebrados (Snow 1971, Givnish 1998, Wang \& Smith 2002).

La ornitocoria fue el síndrome más frecuente en las tres comunidades, lo que coincide con lo reportado por varios autores para las zonas tropicales húmedas (Gentry 1982, Givnish 1998, Martínez-Orea et al. 2009, Buitrón-Jurado \& Ramírez 2014). En los bosques húmedos, los elevados niveles de humedad y nutrientes en el suelo pueden mantener los altos costos de producción de los componentes bioquímicos de la pulpa y los procesos físiológicos para la producción de frutos (Gentry 1982, Milewski 1986), generando una mayor abundancia de recursos y posiblemente de mejor calidad. Lo cual podría incrementar los recursos para aves y otros vertebrados (Gentry 1982, Stiles 1993, Carlo et al. 2004).

Otra hipótesis que se ha planteado para explicar la alta frecuencia de aves en los bosques húmedos tropicales está relacionada con el incremento de insectos herbívoros, los cuales pueden proporcionar "proteína de subsidio" (Snow 1976, Givnish 1999). Muchas aves frugívoras consumen insectos cuando la disponibilidad de frutos disminuye. La abundancia de insectos en los BMM se asocia a la mayor producción de rebrotes en las plantas, estimulada por la alta humedad (Coley 1983). Además, se ha registrado que en los sitios estudiados la producción de hojas en el sotobosque es continua durante todo el año (Williams-Linera 2003), presentándose la mayor abundancia de insectos herbívoros en la época de lluvias (Williams-Linera \& Herrera 2003). Esto coincide con la llegada de aves migratorias en la región centro de Veracruz (Ruelas-Inzunza \& Aguilar-Rodríguez 2010).

El BMM es considerado como uno de los ecosistemas con la mayor diversidad de avifauna (Escalante et al. 1993, Gual-Díaz \& Rendon-Correa 2014), registrándose alrededor de 1,700 especies y altos porcentajes de endemismos (Fjeldsa \& Krabbe 1990, Escalante et al. 1993, Brown \& Kappelle 2001). Particularmente, Veracruz es el estado con el mayor número de especies registradas en este ecosistema (139 spp., Hernández-Baños et al. 1995) y es considerado como corredor migratorio de muchas especies de aves (Pronatura 2013). El incremento en la abundancia de aves migratorias en áreas o rutas de migración influye en la fenología de los frutos (Snow 1971, 1981), la abundancia de aves puede ser un factor de selección natural que favorece a la producción de frutos carnosos (Janzen 1967). Esto podría explicar la importancia de la ornitocoria como síndrome de dispersión predominante en las tres comunidades estudiadas.

En el dosel predominaron diásporas con anemocoria y sinzoocoria, en las tres comunidades estudiadas. Las diásporas con anemocoria utilizan las corrientes de viento para dispersarse; por ello, su ubicación en el dosel favorece este proceso, debido a que la dispersión se incrementa con la velocidad del viento (Givnish 1999, Tackenberg et al. 2003, Nathan et al. 2002). Por otro lado, las diásporas con sinzoocoria (nueces o bellotas) son un recurso alimenticio importante para la fauna por el alto valor nutritivo de sus cotiledones (Vázquez 1998, Rodríguez-Estévez et al. 2007). Estas diásporas pueden ser colectadas directamente de la planta o en el suelo, ofreciendo un recurso alimenticio tanto para animales arborícolas como terrestres.

En la comunidad Cinco Palos la sinzoocoria fue el segundo síndrome de dispersión con mayor número de especies, en la cual las especies del dosel, Oreomunnea mexicana y Quercus spp., aportan el $90 \%$ de área basal de sitio (80.2 $\mathrm{m}^{2} \mathrm{ha}^{-1}$; Velázquez-Escamilla 2016). Estas especies tienen diásporas en forma de nueces con recubrimientos duros (van der Pijl 1972, Howe \& Westley 1997, Perea et al. 2014), características que definen la dispersión por sinzoocoria. La alta densidad de individuos de O. mexicana y Quercus spp. proveen de abundantes recursos alimenticios para pequeños mamíferos y algunas aves (Terborgh et al. 2001 García-Estrada et al. 2002, Guzmán-Guzmán \& Williams-Linera 2006, Johnson et al. 2009, Arroyo-Chacón et al. 2013).

En el Santuario del Bosque de Niebla varias especies del dosel presentaron síndrome anemócoro (11.76\%), entre las que se encuentran Carpinus carolineana y Clethra mexica$n a$, las cuales estructuralmente son importantes, debido a su abundancia y área basal (Velázquez-Escamilla 2016). La alta proporción de especies de plantas que se dispersan por viento se relaciona con la disminución de nutrientes en el suelo y con una mayor apertura del dosel (Ozinga et al. 2004). Esto se debe a que los ambientes con una baja disponibilidad de nutrientes generalmente son bajos y menos densos lo que facilita la dispersión de semillas a través del viento (Ozinga et al. 2004). Sin embargo, para la comunidad estudiada no se conoce con detalle el estatus nutricional de los suelos para determinar su influencia en la presencia de los síndromes anemócoros. Por ello, consideramos que el estado sucesional del Santuario está influyendo en abundancia del síndrome de anemocoria, debido a que $C$. carolineana y $C$. mexicana son especies secundarias que son abundantes en etapas tempranas e intermedias de los BMM del centro de Veracruz (WilliamsLinera et al. 2013).

En el sotobosque los síndromes zoócoros fueron los mejor representados. Esto puede estar relacionado con las condiciones ambientales de mayor humedad y poco viento (Givnish 1999) y la densidad de plantas que ofrecen refugio a los animales (Martínez-Delgado et al. 1996, Cortés et al. 2000, Chad et al.2001, Sobral-Griz \& Machado 2001). En este estudio registramos que el 50 y $60 \%$ de las especies que producen frutos carnosos se encuentran en este estrato, generando abundantes recursos para los dispersores. Además, la frecuencia de plantas con síndrome ornitócoro en el sotobosque puede estar relacionada con la mayor concentración de follaje, lo cual reduce la mortalidad por depredación y enfermedades en las aves (Terborgh 1977). En la Martinica la mamalocoria fue el segundo síndrome más frecuente en el sotobosque. En esta comunidad se han identificado diversos mamíferos pequeños y medianos que consumen frutos 
(Coordinación General del Medio Ambiente 2006), lo que puede estar incrementando la frecuencia de plantas con estos síndromes.

En el Santuario del Bosque de Niebla se registró que en el sotobosque la quiropterocoria tuvo su mejor representación. La dispersión a través de murciélagos frugívoros es un síndrome preferentemente utilizado por plantas que se encuentran en zonas abiertas o con un mayor grado de perturbación, de distintas formas de crecimiento (árboles, arbustos, trepadoras, epífitas y hierbas; Galindo-González et al. 2000, Barbosa-Albuquerque et al. 2006, García-Estrada et al. 2012). En esta comunidad las especies del género Piper fueron más abundantes que en las otras dos comunidades (Velázquez-Escamilla 2016). Estas especies son típicamente dispersadas por murciélagos y generalmente se desarrollan en sitios con mayor perturbación dentro de los BMM (Chazdon et al. 1988). La mayor frecuencia de quiropterocoria en este sitio pueden ser reflejo del estado de conservación en el que se encuentra.

En este trabajo se corroboró que más del $60 \%$ de las plantas clasificadas con el síndrome ornitócoro son forrajeadas por aves. Esto comprueba que la utilización de los síndromes de dispersión a nivel comunidad, ayudan a predecir con alto grado de precisión a los agentes dispersores y dan una idea general sobre la naturaleza de las interacciones, permitiendo conocer parte de la dinámica de estos bosques (Loiselle \& Dirzo 2002, Deckers et al. 2008, Chaín 2005, Buitrón-Jurado \& Ramírez 2014). Sin embargo, es necesario destacar que la identificación de los síndromes tiene un gran valor predictivo, de manera que son útiles como herramienta general de identificación; sin embargo, su determinación no sustituye el estudio del proceso de dispersión (Howe \& Smallwood 1982).

La frecuencia e importancia de los síndromes de dispersión en los bosques mesófilos de montaña cambian en función de la estructura (abundancia y estratos) y composición de la vegetación. El síndrome de dispersión predominante fue la ornitocoria, revelando la importancia de las aves como principal agente de dispersión. El síndrome ornitócoro ayuda a describir a los dispersores potenciales de las especies vegetales en las comunidades estudiadas. Por ello, es relevante entender de manera puntual el impacto de la dispersión de semillas de este grupo de vertebrados y su influencia en los patrones de reclutamiento, distribución y abundancia de las plantas en los BMM. En particular, esta información es necesaria para establecer estrategias de conservación, restauración y manejo sostenible de estos bosques, los cuales son uno de los ecosistemas en mayor riesgo a nivel mundial (Levin \& Murrel 2003, Hamilton et al. 1995, Hernández-Ladrón de Guevara et al. 2012).

\section{Agradecimientos}

Los autores agradecen el apoyo de G. Torres Martínez e I. Martínez González en el trabajo de campo. Este proyecto fue financiado por el proyecto PROMEP/103.5/13/9126. El CONACyT otorgó una beca para estudios de maestría a T.L. Velázquez Escamilla.

\section{Literatura citada}

Armesto JJ, Rozzi R. 1989. Seed dispersal syndromes in the rain forest of Chiloé: evidence for the importance of biotic dispersal in a temperate rain forest. Journal Biogeography 16: 219-226. DOI: https://doi.org/10.2307/2845258

Arroyo-Chacón E, Riechers-Pérez A, Naranjo EJ. Rivera-Velázquez G. 2013. Riqueza, abundancia y diversidad de mamíferos silvestres entre hábitats en el Parque Nacional Cañón del Sumidero, Chiapas, México. Therya 3: 647-676. DOI: https://doi.org/10.12933/therya-13-140

Baltazar-Hernández S. 2014. La importancia de la dispersión de semillas en la recuperación del bosque mesófilo de montaña del centro de Veracruz, México. MSc. Thesis, Instituto de Ecología, AC.

Barbosa-Albuquerque L, Velázquez A, Mayorga-Saucedo R. 2006. Solanaceae composition, pollination and seed dispersal syndromes in Mexican Mountain Cloud Forest. Acta Botanica Brasilica 3: 599-613.

DOI: http://dx.doi.org/10.1590/S0102-33062006000300010

Brown AD, Kappelle M. 2001. Introducción a los bosques nublados del Neotrópico: una síntesis regional. In: Kapelle M, Brown AD, eds. Bosques Nublados del Neotrópico. Santo Domingo de Heredia: INBIO, pp. 25-40. ISBN-13: 9789968702508

Bruijnzeel LA, Scatena FN, Hamilton LS. 2010. Tropical Montane Cloud Forests: Science for Conservation and Management. New York: Cambridge University Press. ISBN-13: 978-0521760355

Buitrón-Jurado G, Ramírez N. 2014. Dispersal spectra, diaspore size and the importance of endozoochory in the equatorial Andean montane forest. Flora Morphology, Dristribution, Functional Ecology Plants 209: 299-311. DOI: https://doi.org/10.1016/j.flora.2014.03.009

Carlo TA, Morales JM. 2016. Generalist birds promote tropical forest regeneration and increase plant diversity via rare-biased seed dispersal. Ecology 97: 1819-1831.

DOI: https://doi.org/10.1890/15-2147.1

Carlo TA, Collazo JA, Groom MJ. 2004. Influences of fruit diversity and abundance on bird use of two shaded coffee plantations. Biotropica 36: 602-614. DOI: https://doi.org/10.1111/j.1744-7429.2004.tb00354.x

Cavieres LA, Papic C, Castor C. 1999. Variación altitudinal en los síndromes de dispersión de semillas de la vegetación andina de la cuenca del río Molina, Chile Central $\left(33^{\circ} \mathrm{S}\right)$. Gayana Botanica 56: 115-123.

Chad EL, Clark TW, Derting TL. 2001. Food selection by the white-footed mouse (Peromyscus leucopus) on the basis of energy and protein contents. Canadian Journal of Zoology 79: 562- 568. DOI: https://doi.org/10.1139/z01-015

Chaín GA. 2005. Síndromes de Dispersión en el Mosaico Vegetacional de la Región de Nizada (Oaxaca), México. BSc Thesis. Universidad Nacional Autónoma de México.

Chapman H, Cordeiro NJ, Dutton P, Wenny D, Kitamura S, Kaplin B, Melo FPL, Lawes MJ. 2016. Seed-dispersal ecology of tropical montane forest. Journal of Tropical Ecology 32: 1-18. DOI: https://doi.org/10.1017/S0266467416000389 
Chazdon RL, Williams K, Field CB. 1988. Interactions between crown structure and light environment in five rain forest Piper species. American Journal of Botany 75: 1459-1471. DOI: https://doi.org/10.2307/2444696

Coley PD. 1983. Herbivory and defensive characteristics of tree species in a lowland tropical forest. Ecology Monographs $\mathbf{5 3}$ : 209-234. DOI: https://doi.org/10.2307/1942495

Coordinación General del Medio Ambiente. 2006. Programa de Manejo del Área Natural "La Martinica" Banderilla, Veracruz. Veracruz: Secretaría de Desarrollo Social y Medio Ambiente, Gobierno del Estado de Veracruz.

Cortés A, Rosenmann M, Bozinovic F. 2000. Water economy in rodents: evaporative water loss and metabolic water production. Revista Chilena de Historia Natural 73: 311-321. DOI: http://dx.doi.org/10.4067/S0716-078X2000000200006

Dalling J. 2002. Ecología de semillas. In: Guariguata MR, Kattan GH, eds. Ecología y Conservación de Bosques Neotropicales. Costa Rica: Asociación de Editoriales Universitarias de América Latina y el Caribe, pp. 345-375. ISBN-13: 978-9968801119

Deckers B, Verheyen K, Vanhellemont M, Maddens E, Muys B, Hermy M. 2008. Impact of avian frugivores on dispersal and recruitment of the invasive Prunus serotina in an agricultural landscape. Biological Invasions 10: 717-727. DOI: https://doi.org/10.1007/s10530-007-9164-3

Dormann CF. 2011. How to be a specialist? Quantifying specialisation in pollination networks. Network Biology 1: 1-20.

Dormann CF, Gruber B, Fruend J. 2008. Introducing the bipartite Package: Analysing Ecological Networks. $R$ news 8 : 8-11.

Escalante P, Navarro-Sigüenza AG, Peterson AT. 1993. A geographic, ecological and historical analysis of land bird diversity in Mexico. In: Ramamoorthy TP, Bye R, Lot A, Fa J, eds. Biological Diversity of Mexico: Origins and Distributions. New York: Oxford University Press, 279-304. ISBN-13: 978-0195066746

Fischer KE, Chapman CA. 1993. Frugivores and fruit syndromes: differences in patterns at the genus and species level. Oikos 66: 472-482. DOI: https://doi.org/10.2307/3544942

Fjeldsa J, Krabbe N. 1990. Birds of the high Andes. Museum Tusculanum. Chicago: University of Chicago Press. ISBN13: 978-8788757163

Galindo-González J, Guevara S, Sosa VJ. 2000. Bat- and birdgenerated seed rains at isolated trees in pastures in a tropical rainforest. Conservation Biology 14: 1963-1703. DOI: https://doi.org/10.1111/j.1523-1739.2000.99072.x

Ganesh T, Davidar P. 2001. Dispersal mode of tree species in the wet forest of southern Western Ghats. Current Science 80: 394-399.

García-Estrada C, Damon A, Sánchez-Hernández C, Soto-Pinto L, Ibarra-Núñez G. 2012. Diets of frugivorous bats in montane rain forest and coffee plantations in southeastern Chiapas, México. Biotropica 44: 394-401.

DOI: https://doi.org/10.1111/j.1744-7429.2011.00816.x

Gentry AH. 1982. Patterns of Neotropical Plant Species Diversity. In: Hecht MK, Wallace B, Prance GT, eds. Evolutionary Biology. Boston: Springer, 1-84.

DOI; https://doi.org/10.1007/978-1-4615-6968-8 1
Gentry AH. 1995. Patterns of diversity and floristic composition in neotropical montane forest. In: Churchill SP, Balslev H, Forero E, Luteyn JL, eds. Biodiversity and Conservation of Neotropical Montane Forest. New York: The New York Botanical Garden, 89-102. ISBN-13: 978-0893274009

Givnish TJ. 1998. Adaptative plant evolution on islands: classical patterns, molecular data, new insights. In: Grant P, ed. Evolution on Islands. Oxford: Oxford University Press, 281304. ISBN-13: 978-0198501725

Givnish TJ. 1999. On the causes of gradients in tropical tree diversity. Journal of Ecology 87:193-210. DOI: https://doi.org/10.1046/j.1365-2745.1999.00333.x

Gual-Díaz M, Rendón-Correa A. 2014. Bosques Mesófilos de Montaña: Diversidad, Ecología y Manejo. México DF: Comisión Nacional para el Conocimiento y Uso de la Biodiversidad. ISBN: 978-607-8328-07-9

Guariguata MR, Kattan GH. 2002. Ecología y Conservación de Bosques Neotropicales. Costa Rica: Asociación de Editoriales Universitarias de América Latina y el Caribe,. ISBN-13: 978-9968801119

Guzmán-Guzmán J, Williams-Linera G. 2006. Edge effect on acorn removal and oak seedling survival in Mexican lower montane forest fragments. New Forests 31: 487-495. DOI: https://doi.org/10.1007/s11056-005-2015-0

Hamilton LS, Juvik JO, Scatena FN. 1995. Tropical Montane Cloud Forests. New York: Springer-Verlag. eBook ISBN: 978-1-4612-2500-3; DOI 10.1007/978-1-4612-2500-3

Hampe A, Bairlein F. 2000. Modified dispersal-related traits in disjunct populations of bird-dispersed Frangula alnus (Rhamnaceae): a result of its Quaternary distribution shifts? Ecography 23: 603-613. DOI: https://doi.org/10.1111/j.1600-0587.2000.tb00179.x

Hernández-Baños B, Peterson AT, Navarro-Sigüenza AG, Escalante-Pliego P. 1995. Bird faunas of the humid montane forests of Mesoamerica: biogeographic patterns and priorities for conservation. Bird Conservation International 5: 251277. DOI: https://doi.org/10.1017/S0959270900001039

Hernández-Ladrón de Guevara I, Rojas-Soto OR, López-Barrera F, Puebla-Olivares F, Díaz-Castelazo C. 2012. Dispersión de semillas por aves en un paisaje de bosque mesófilo en el centro de Veracruz, México: su papel en la restauración pasiva. Revista Chilena de Historia Natural 85: 89-100. DOI: http://dx.doi.org/10.4067/S0716-078X2012000100007

Herrera CM. 1987. Vertebrate-dispersed plants of the Iberian Peninsula-a study of fruit characteristics. Ecological Monographs 57:305-331. DOI: https://doi.org/10.2307/2937089

Herrera CM. 1989. Seed dispersal by animals: a role in angiosperm diversification? The American Naturalist 133: 309-322. DOI: https://doi.org/10.1086/284921

Herrera CM. 1992. Interspecific variation in fruit shape: allometry, phylogeny, and adaptation to dispersal agents. Ecology 73: 1832-1841. DOI: https://doi.org/10.2307/1940034

Howe HF, Smallwood J. 1982. Ecology of seed dispersal. Annual Review of Ecology and Systematics 13: 201-28. DOI: https://doi.org/10.1146/annurev.es.13.110182.001221

Howe HF, Westley LC. 1997. Ecology of pollination and seed dispersal. In: Crawley MJ, ed. Plant Ecology. Oxford: Blackwell Science, 262-283. ISBN-13: 978-0632036394 
Janzen DH. 1967. Synchronization of sexual reproduction of trees within the dry season in Central America. Evolution 21: 620-637. DOI: https://doi.org/10.2307/2406621

Johnson PS, Shifley SR, Rogers R. 2009. The ecology and silviculture of oaks. Wallingford, UK: CABI Publishing. ISBN13: 978-1845934743

Jordano P. 1995. Angiosperm fleshy fruits and seed dispersers- a comparative analysis of adaptation and constraints in plantsanimal interactions. The American Naturalist 145:163-191. DOI: https://doi.org/10.1086/285735

Jordano P. 2000. Fruits and frugivory. In: Fenner M, ed, Seeds: The Ecology of Regeneration in Plant Communities. Wallingford, UK: CABI Publishing, pp.125-162. ISBN-13: 9780851994321

Kuhlmann M, Ribeiro JF. 2016. Evolution of seed dispersal in the Cerrado biome: ecological and phylogenetic considerations. Acta Botanica Brasilica 30: 271-282.

DOI: https://doi.org/10.1590/0102-33062015abb0331

Levin SA, Murrell DJ. 2003.The community-level consequences of seed dispersal patterns. Annual Review of Ecology, Evolution and Systematic 34: 549-574.

DOI: https://doi.org/10.1146/annurev.ecolsys.34.011802.132 400

Levin SA, Muller-Landau HC, Nathan R, Chave J. 2003. The ecology and evolution of seed dispersal: a theoretical perspective. Annual Review of Ecology, Evolution and Systematics, 34: 575-604.

DOI: https://doi.org/10.1146/annurev.ecolsys.34.011802.132 428

Loiselle B, Dirzo R. 2002. Plant-animal interactions and community structure. In: Chazdon RL, Whitmore TC, eds. Foundations of Tropical Forest Biology: Classic Papers with Commentaries. Chicago: University of Chicago Press, 269338. ISBN-13: 978-0226102252

Lomáscolo SB, Speranza P, Kimball RT. 2008. Correlated evolution of fig size and color supports the dispersal syndromes hypothesis. Oecologia 156: 783-796.

DOI: https://doi.org/10.1007/s00442-008-1023-0

López M, Ramírez N. 1998. Síndromes de dispersión de diásporas de una comunidad arbustiva de la Guayana Venezolana. Ecotrópicos 11: 15-32.

Lord JM, Markey AS, Marshall J. 2002. Have frugivores influenced the evolution of fruit traits in New Zealand? In: Levey DJ, Silva WR, Galetti M, eds. Seed Dispersal and Frugivory: Ecology, Evolution and Conservation. Wallingford, UK: CABI Publishing, 55-68. ISBN-13: 978-0851995250

Lord JM. 2004. Frugivore gape size and the evolution of fruit size and shape in southern hemisphere floras. Austral Ecology 29: 430-436.

DOI: https://doi.org/10.1111/j.1442-9993.2004.01382.x

Luna-Monterrojo VE. 1997. Estudio de vegetación y flora del municipio de Coatepec, Veracruz. Tesis de Licenciatura, Facultad de Biología, Universidad Veracruzana, Xalapa, Ver.

Martínez-Delgado E, Mellink E, Aguirre-Rivera JR, GarcíaMoya E. 1996. Removal of piñon seeds by birds and rodents in San Luis Potosí México. The Southwestern Naturalist 41: 270-274.
Martínez-Orea Y, Castillo-Argüero S, Guadarrama-Chávez P. 2009. La dispersión de frutos y semillas y la dinámica de comunidades. Ciencias 96: 38-41.

Milewski AV. 1986. A comparison of bird-plant relationships in southern Australia and southern Africa. In: Ford HA, Paton DC, eds. The Dynamic Partnership: Birds and Plants in Southern Africa. Adelaide: Information Sa, pp. 111-118. ISBN: 9780724346400

Moles AT, Ackerly DD, Tweddle JC, Dickie JB, Smith R, Leishman MR, Mayfield MM, Pitman A, Wood JT, Westoby M. 2007. Global patterns in seed size. Global Ecology and Biogeography 16: 109-116. DOI: https://doi.org/10.1111/j.1466-8238.2006.00259.x

Nathan R, Katul G, Horn HS, Thomas SM, Oren R, Avissar R, Pacala SW, Levin SA. 2002. Mechanisms of long- distance dispersal of seeds by wind. Nature 418: 409-413. DOI: https://doi.org/10.1038/nature00844

Ozinga WA, Bekker RM, Schaminée JH, Van Groenendael JM. 2004. Dispersal potential in plant communities depends on environmental conditions. Journal of Ecology 92: 767-777. DOI: https://doi.org/10.1111/j.0022-0477.2004.00916.x

Perea R, San Miguel A, Gil L. 2014. Interacciones planta- animal en la regeneración de Quercus pyrenaica; ecología y gestión. Ecosistemas 23: 18-26.

DOI: https://doi.org/10.7818/ECOS.2014.23-2.04

PRONATURA. 2013. Aves migratorias en su travesía por México. http://www.pronatura-sur.org/web/nota.php?id=30 (acceso febrero 15,2018$)$.

Rodríguez-Estévez V, García A, Perea J, Mata C, Gómez AG. 2007. Producción de bellotas en la Dehesa: factores influyentes. Archivos de Zootecnia 56: 25-43.

Rosas-Guerrero V, Aguilar R, Martén-Rodríguez S, Asworht L, Lopezaraiza-Mikel M, Bastida JM, Quesada M. 2014. A quantitative review of pollination syndromes: do floral traits predict effective pollinators? Ecology Letters 17: 388-400. DOI: https://doi.org/10.1111/ele.12224

R Core Team. 2018. R, a language and environment for statistical computing. R Foundation for Statistical Computing, Vienna. http://www.R-project.org (acceso marzo 15, 2018).

Ruelas-Inzunza E, Aguilar-Rodríguez SH. 2010. La avifauna urbana del parque ecológico Macuiltépetl en Xalapa, Veracruz, México. Ornitología Neotropical 21: 87-103.

Scatena F, Bruijnzeel L, Bubb P. 2010. Setting the stage. In: Bruijnzeel LA, Scatena FN, Hamilton LS, eds. Tropical Montane Cloud Forests: Science for Conservation and Management. New York: Cambridge University Press, pp 3-13. ISBN-13: 978-0521760355

Schemske DW, Mittelbach GC, Cornell HV, Sobel JM, Roy K. 2009. Is there a latitudinal gradient in the importance of biotic interactions? Annual Review of Ecology, Evolution and Systematics 40: 245-269. DOI: https://doi.org/10.1146/annurev.ecolsys.39.110707.173430

Sinnott-Armstrong MA, Downie AE, Federman S, Valido A, Jordano P, Donoghue MJ. 2018. Global geographic patterns in the colours and sizes of animal-dispersed fruits. Global Ecology and Bigeography 27: 1339-1351.

DOI: https://doi.org/10.1111/geb.12801 
Snow DW. 1976. The Web of Adaptation: Bird Studies in the American Tropics. New York: Cornell University Press. ISBN-13: 978-0801493164

Snow D. 1981. Tropical Frugivorous birds and their food plants: a world survey. Biotropica 13: 1-14.

DOI: https://doi.org/10.2307/2387865

Snow DW. 1971. Evolutionary aspects of fruit eating by birds. Ibis 113: 194-202. DOI: https://doi.org/10.1111/j.1474-919X.1971.tb05144.x

Sobral-Griz LM, Machado ICS. 2001. Fruiting phenology and seed dispersal syndromes in caatinga, a tropical dry forest in the northeast of Brazil. Journal of Tropical Ecology 17: 303321. DOI: https://doi.org/10.1017/S0266467401001201

Stiles EW. 1993.The influence of pulp lipids on fruit preference by birds. Vegetatio 107: 227-235.

Tackenberg O, Poschlod P, Boon S. 2003. Assessment of wind dispersal potential in plant species. Ecological Monographs 73:191-205.

DOI: https://doi.org/10.1890/0012-9615(2003)073[0191:AO WDPI]2.0.CO;2

Terborgh J, Lopez L, Nuñez P, Rao M, Shahabuddin G, Orihuela G, Riveros M, Ascanio R, Adler GH, Lambert TD, Balbas L. 2001. Ecological Meltdown in predator-Free Forest Fragments Science 294: 1923-1926.

DOI: https://doi.org/10.1126/science.1064397

Terborgh J. 1977. Bird species diversity on an Andean elevation gradient. Ecology 58: 1007-1019. DOI: https://doi.org/10.2307/1936921

Toledo-Garibaldi T, Williams-Linera G. 2014. Tree diversity patterns in successive vegetation types along an elevation gradient in the Mountains of Eastern Mexico. Ecological Research 29: 1097-1104.

DOI: https://doi.org/10.1007/s11284-014-1196-4

van der Pijl L. 1972. Principles of Dispersal in Higher Plants. New York: Springer-Verlag. eBook ISBN: 978-3-642-961083; DOI: 10.1007/978-3-642-96108-3

Vázquez FM. 1998. Semillas de Quercus: biología ecología y manejo. España: Publicaciones de la Secretaría General Técnica, Consejería de Agricultura y Comercio. ISBN: 848107-028-9

\section{Editor de sección: Neptalí Ramírez-Marcial}

Authors' contributions: TLVE concibió, diseñó el trabajo, desarrolló el trabajo de campo y gabinete, realizó el análisis estadístico y escribió el documento. CDC diseñó el trabajo, apoyo en los análisis estadísticos y revisó el documento. BRG apoyó los análisis estadísticos y revisó el documento. NVR (http://orcid.org/0000-00027768-2279) concibió, diseñó el trabajo, apoyó el trabajo de campo y gabinete, escribió el documento.
Vázquez GA, Givnish T. 1998. Altitudinal gradients in tropical forest composition, structure, and diversity in the Sierra de Manantlán. Journal of Ecology 86: 999-1020. DOI: https://doi.org/10.1046/j.1365-2745.1998.00325.x

Velázquez-Escamilla TL. 2016. Síndromes de dispersión en tres comunidades de bosque mesófilo de montaña. MSc. Thesis, Universidad Veracruzana.

Velázquez-Rosas N, Meave J, Vázquez-Santana S. 2002. Elevational variation of leaf traits in montane rain forest tree species at La Chinantla, southern México. Biotropica 34: 534-546.

DOI: https://doi.org/10.1111/j.1744-7429.2002.tb00572.x

Wang BC, Smith TB. 2002. Closing the seed dispersal loop. Trends in Ecology and Evolution 17: 379-385.

DOI: https://doi.org/10.1016/S0169-5347(02)02541-710.101 6/S0169-5347(02)02541-7

Wheelwright NT, Haber WA, Murray KG, Guindon C. 1984. Tropical fruit-eating birds and their food plants: a survey of a Costa Rican lower montane forest. Biotropica 16: 173-192. DOI: https://doi.org/10.2307/2388051

Williams-Linera G. 2003. Temporal and spatial phenological variation of understory shrubs in a tropical montane cloud forest. Biotropica 35: 28-36. DOI: https://doi.org/10.1111/j.1744-7429.2003.tb00259.x

Williams-Linera G. 2012. El bosque de niebla del Centro de Veracruz: ecología, historia y destino en tiempos de fragmentación y cambio climático. Xalapa, Veracruz: Comisión Nacional para el Conocimiento y Uso de la Biodiversidad, Instituto de Ecología, AC. ISBN: 9707091010. http://www. inecol.edu.mx/librobosquedeniebla.pdf (consultado en nmarzo de 2017).

Williams-Linera G, Herrera F. 2003. Folivory, herbivores and environment in the understory of a tropical montane cloud forest. Biotropica 35: 67-73.

DOI: https://doi.org/10.1111/j.1744-7429.2003.tb00263.x

Williams-Linera G. Toledo-Garibaldi M, Gallardo-Hernández C. 2013. How heterogeneous are the cloud forest communities in the mountains of central Veracruz, México? Plant Ecology 214: 685-701.

DOI: https://doi.org/10.1007/s11258-013-0199-5 